\title{
Modeling and Simulation of RLS for Adaptive Noise Cancellation Hu Zhongyu ${ }^{1 a^{*}}$, Yue Qiang ${ }^{2 b}$,Chen Weiming ${ }^{1 c}$
}

\author{
${ }^{1}$ School of Auto-control and Mechanical Engineering, Kunming University, Kunming 650214,China \\ ${ }^{2}$ School of Information Technology, Kunming University, Kunming 650214,China; \\ apoundblue@126.com, bwallay@126.com, ${ }^{\mathrm{c}} 239336262 @ q q . c o m$
}

Keywords: adaptive noise cancellation ; RLS algorithm ; MATLAB simulation.

\begin{abstract}
In this paper, an adaptive noise cancellation system based on RLS algorithm is presented, and the principle of noise cancellation system and the steps and processes of RLS algorithm are described. The simulation of adaptive noise cancellation system based on RLS algorithm is obtained by using an example. The simulation results show that the RLS adaptive filter can effectively eliminate the interference and get useful signal in two cases of single frequency interference and broadband noise. It is proved that the adaptive noise cancellation system based on RLS algorithm can eliminate the noise and improve the signal quality of speech communication in the communication processing of speech signal.
\end{abstract}

Keywords:

\section{Introduction}

The adaptive noise cancellation system is widely used in modern communication signal processing. For example, in the process of high altitude flight, the pilot can keep in touch with the ground navigation station in real time. In the process of communication, the pilot's voice is transmitted to the navigation station. Such a variety of environmental noise will have a great impact on communication. When the noise is very large, the useful speech signal will be drowned by the noise, which will seriously affect the communication. It can install a microphone to receive all kinds of noise in the engine room in the cabin, the signal and speech signal at the same time back to the ground. Then, the adaptive noise cancellation system is used to deal with the speech signal to eliminate the noise in the speech, so as to improve the communication quality. The existing research adaptive noise cancellation system is based on the LMS algorithm, which is effective in the environmental noise is a stable random signal. When the environmental noise is not stationary random signal, the LMS algorithm is very difficult to track the changes of the statistical characteristics of the noise disturbance, and thus the convergence effect is generally. In this paper, the adaptive noise cancellation system based on RLS algorithm overcomes the above shortcomings, and can achieve more satisfactory results in non-stationary environment. [1] [2]

\section{Algorithm formulation}

\subsection{Adaptive Filters}

In this section we first go through an examination of the filter structure with an emphasis on FIR(finite impulse responses)filters. This is followed by a review of the wiener filter leading to the development of the LMS(least mean aquares)algorithm.

A noise canceller is a closed loop linear adaptive filter used for direct system modeling. There are many different combinations of filters and algorithms, depending on the particular application requirements; from FIR to IIR (infinite impulse response) filters, from LMS to RLS (recursive least squares) algorithms. For noise cancellation, there is a classical standard adaptive filter formation. The filter part is made up of the most commonly used structure: A FIR filter which is also known as a tapped delay line, non-recursive or feed-forward transversal filter,as shown in Fig.1. In this paper we will only consider RLS filters for noise cancellation. 


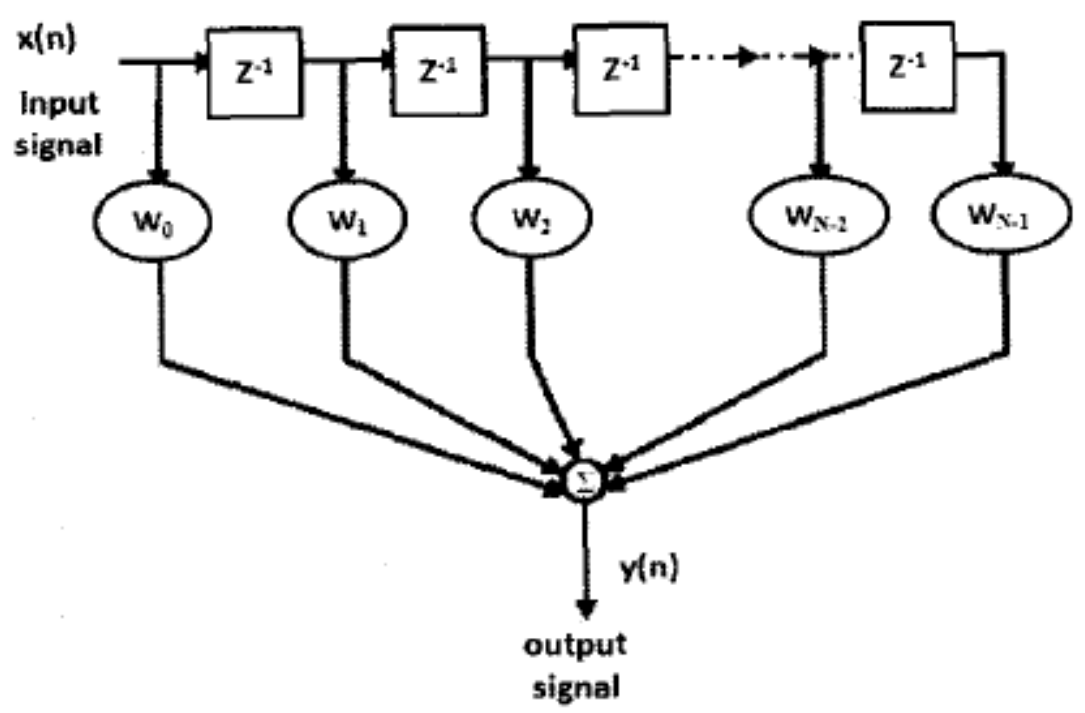

Fig.1 FIR filter structure

2.2 Adaptive noise cancellation system

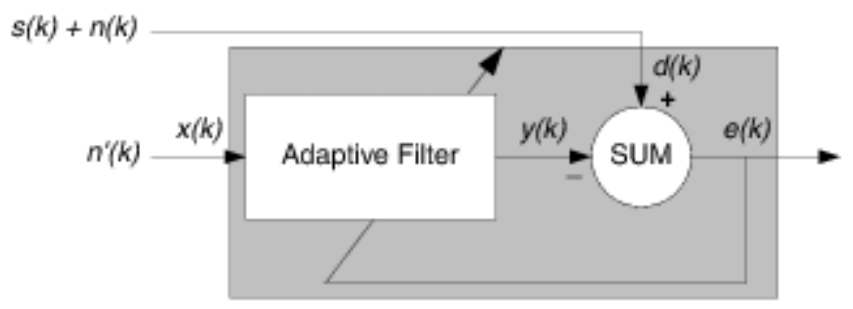

Fig.2 Block diagram of adaptive noise cancellation system

In Fig 2, the original input signal D (n) includes a useful signal s (k) and noise $n(k)$. The reference input signal $\mathrm{y}(\mathrm{k})$ is a signal associated with the noise $\mathrm{n}(\mathrm{k})$. The reference signal is changed to y $(\mathrm{k})$ after the adaptive filter. The output of the adaptive noise cancellation system is:

To the Eq.1 on both sides of the square:

$$
\mathrm{e}(\mathrm{k})=\mathrm{s}(\mathrm{k})+\mathrm{n}(\mathrm{k})-\mathrm{y}(k)
$$

$$
\mathrm{e}^{2}(\mathrm{k})=\mathrm{s}^{2}(\mathrm{k})+[\mathrm{n}(\mathrm{k})-\mathrm{y}(k)]^{2}+2 s(k)[n(k)-\mathrm{y}(k)]
$$

On the Eq.2 to take mathematical expectation:

$$
E\left[\mathrm{e}^{2}(\mathrm{k})\right]=\mathrm{E}\left[\mathrm{s}^{2}(\mathrm{k})\right]+\mathrm{E}[\mathrm{n}(\mathrm{k})-\mathrm{y}(k)]^{2}
$$

Assuming here that $\mathrm{s}(\mathrm{k}), \mathrm{n}(\mathrm{k})$ and $\mathrm{y}(\mathrm{k})$ are uncorrelated and statistical stability. As $\mathrm{E}\left[\mathrm{s}^{2}(\mathrm{k})\right]$ is not related to the regulation of the filter, if the adaptive filter makes the $E\left[\mathrm{e}^{2}(\mathrm{k})\right]$ is the smallest, and that is $E[n(k)-y(k)]^{2}$ is the smallest. As the Eq.1, with $E[n(k)-y(k)]^{2}$ become the smallest, $E[e(k)-s(k)]$ is the smallest. The mean square deviation of e $(\mathrm{k})$ and $\mathrm{s}(\mathrm{k})$ is smallest, In the ideal case, $\mathrm{n}(\mathrm{k})=\mathrm{y}(\mathrm{k})$, $\mathrm{e}(\mathrm{k})=\mathrm{s}(\mathrm{k})$, then, the reference signal is transformed into $\mathrm{n}(\mathrm{k})$ by the adaptive filter, then the $\mathrm{s}(\mathrm{k})$ is retained after the original input minus $\mathrm{n}(\mathrm{k})$. [3] [4]

\subsection{RLS algorithm}

RLS algorithm is the recursive least squares algorithm. [5] [6]The basic idea of RLS algorithm is to estimate the number of taps of the k-1 sub iteration filter, and to calculate the latest estimates based on the newly arrived data. Recursive least squares algorithm using the average minimization two squares, and even the minimum square error.

We then apply the following modified normalized RLS algorithnm which tracks the desired solution

$$
\begin{gathered}
\mathrm{y}(\mathrm{k})=\widehat{w}(k-1) \cdot x(k) \\
\widehat{w}(k+1)=\widehat{w}(k)+k(k)^{H} e(k) \\
k(k)=\frac{\mu^{-1} p(k-1) X(k)}{1+\mu^{-1} X(k)^{H} p(k-1) X(k)} \\
p(k)=\mu^{-1} p(k-1)-\mu^{-1} k(k) x(k)^{H} p(k-1)
\end{gathered}
$$


Where $\mu^{-1}$ denotes the reciprocal of the exponential weighting factor.

\section{Simulations}

Here, we will illustrate the ability of the RLS filter to extract useful information from a noisy signal. The information bearing signal is a sine wave that is corrupted by additive white gaussian noise.

The adaptive noise cancellation system assumes the use of two microphones. A primary microphone picks up the noisy input signal, while a secondary microphone receives noise that is uncorrelated to the information bearing signal, but is correlated to the noise picked up by the primary microphone.

In the following graphic: Fig.3,we define the information bearing signal is a sine wave of 0.1 cycles/sample. The noise picked up by the secondary microphone is the input for the RLS adaptive filter. The noise that corrupts the sine wave is a lowpass filtered version of (correlated to) this noise. The sum of the filtered noise and the information bearing signal is the desired signal for the adaptive filter.

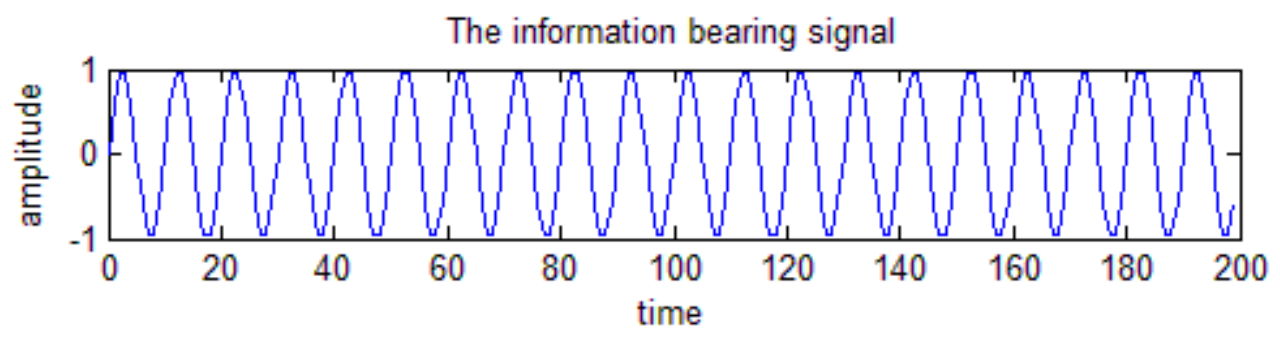

Noise picked up by the secondary microphone

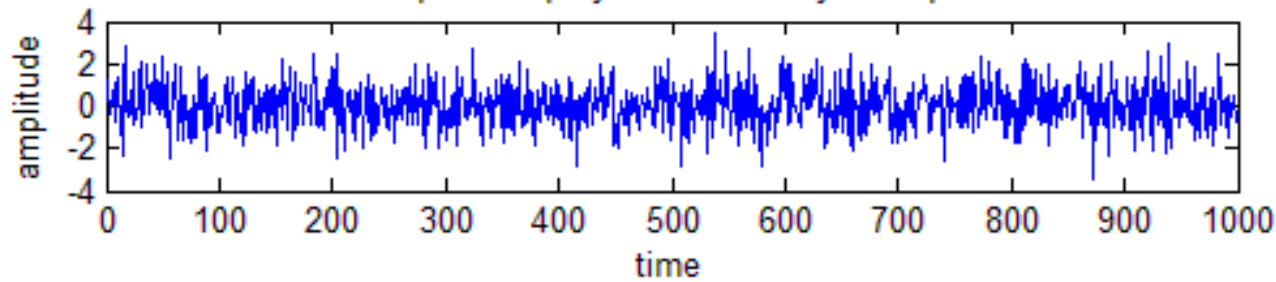

Desired input to the Adaptive Filter $=$ Signal + Filtered Noise

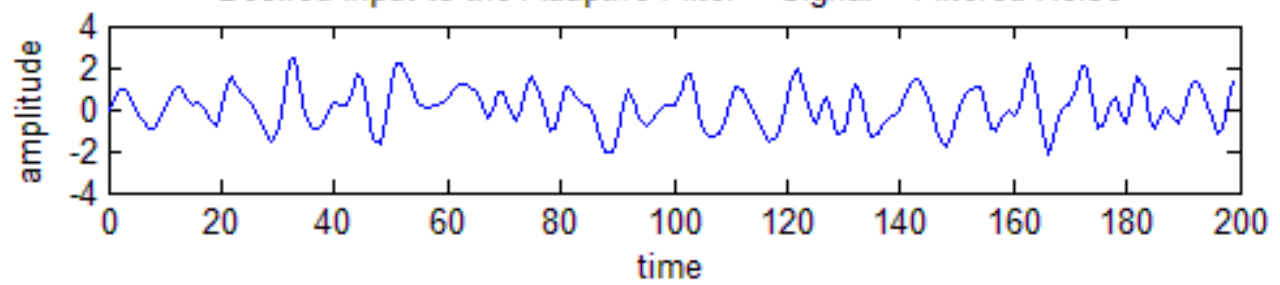

Fig.3 time domain waveform of $\mathrm{s}(\mathrm{k}), \mathrm{n}(\mathrm{k}), \mathrm{d}(\mathrm{k})$

Set and initialize RLS adaptive filter parameters and values as Tab.1.

Table 1 RLS adaptive filter parameters and values

\begin{tabular}{|l|l|l|}
\hline parameters & values & Explain \\
\hline M & 32 & Filter order \\
\hline lam & 1 & Exponential weighting factor \\
\hline delta & 0.1 & Initial input covariance estimate \\
\hline w0 & zeros(M,1) & Initial tap weight vector \\
\hline P0 & $(1 /$ delta)*eye(M,M) & Initial setting for the P matrix \\
\hline Zi & zeros(M-1,1) & FIR filter initial states \\
\hline
\end{tabular}

Running the RLS adaptive filter for 1000 iterations. Fig. 4 shows the convergence of the adaptive filter response to the response of the FIR filter. 


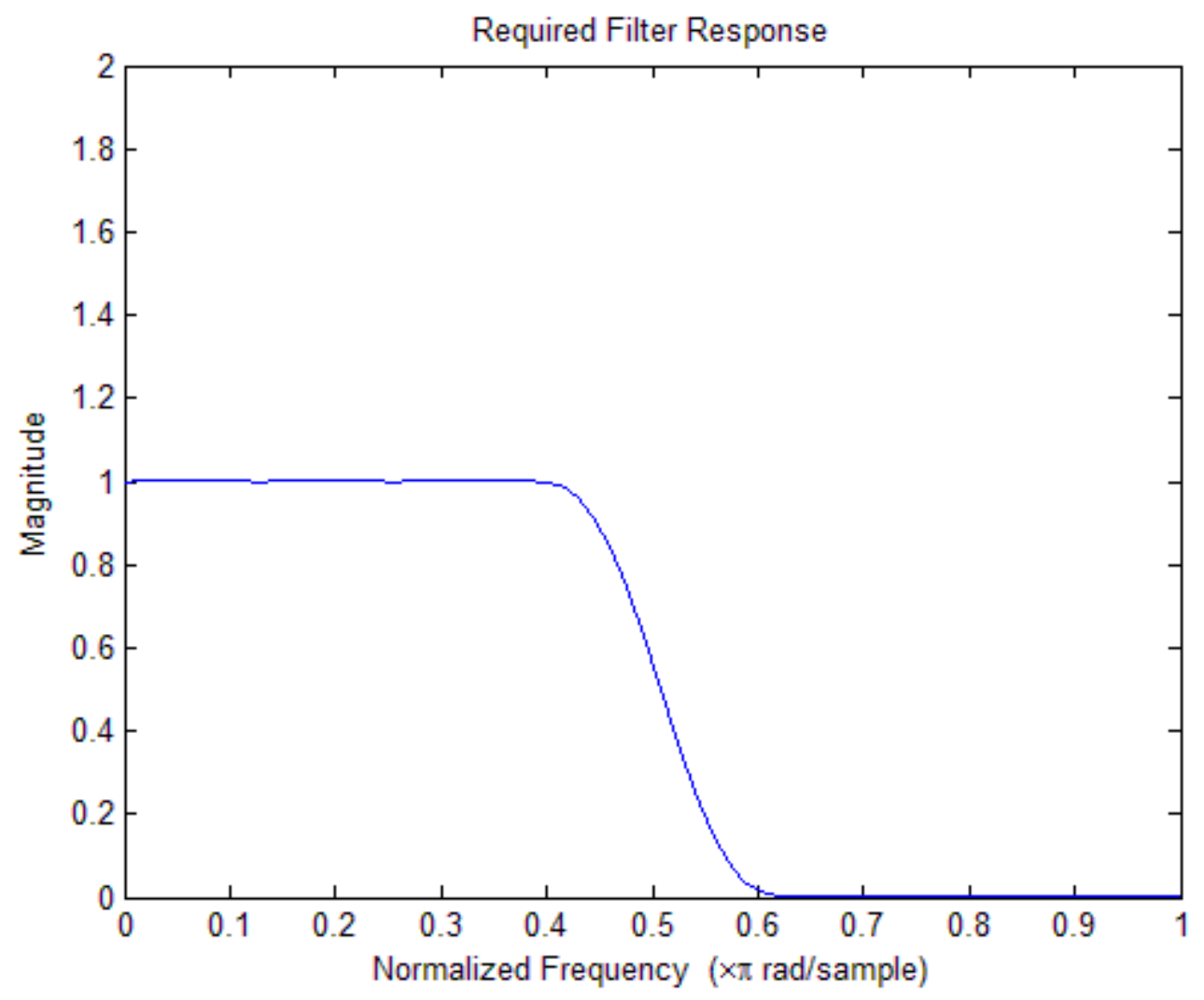

Fig. 4 the response of the FIR filter.

As the adaptive filter converges, the filtered noise should be completely subtracted from the "signal + noise" signal and the error signal 'e' should contain only the original signal.
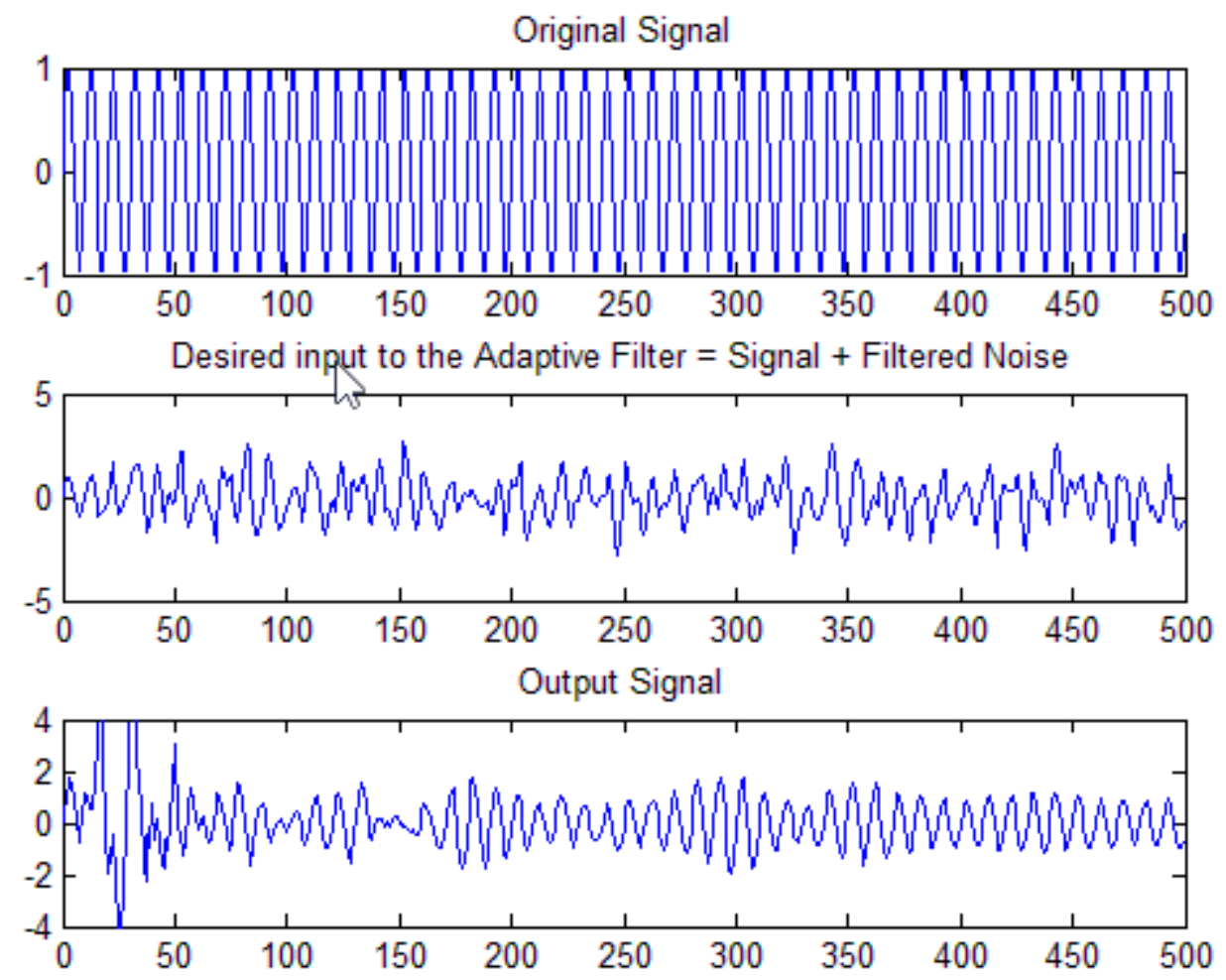

Fig.5 Comparison of three signals

Fig. 5 is showing that the output signal of the original input signal is basically consistent with the useful signal after the RLS filter, which means that the RLS adaptive noise cancellation system 
eliminates the noise in the original input. Meanwhile,the convergence speed of RLS algorithm is fast, and the performance of RLS algorithm is proved to be superior.

\section{Conclusions}

The adaptive noise cancellation system based on RLS algorithm is studied in this paper. The principle of the adaptive noise cancellation system is described and the RLS algorithm is derived. Computer simulation is carried out by MATLAB. The simulation results confirm the feasibility of the RLS algorithm, which indicates that the RLS adaptive noise cancellation system can improve the quality of the speech signal in the communication. Figures.

\section{Acknowledgement}

In this paper, the research was sponsored by the Science Research Foundation Project of the Education Department of Yunnan Province (Project No. 2011Y237), Science Research Project of Kunming University (Project No. XJL15013) and Science Research Project of Kunming University (Project No. XJL14006).

\section{References}

[1] S. Haykin, Adaptive Filter Theory, Prentice-Hall, Englewood Cliffs, New Jersey, 1996.

[2] J. Homer, R.R. Bitmead, I. Mareels, Quantifying the effects of dimension on the convergence rate of the LMS adaptive FIR estimator, IEEE Trans. Signal Process. 46 (10) (Oct. 1998) 2611-2615.

[3] [1] C.H. Knapp, G.C. Carter, The generalized correlation method forestimation of time delay, IEEE Trans. Acoust., Speech, Signal Process.24 (4) (1976) 320-327.

[4] J. Yang. Study on Signal Analyzing and Processing for Leak Detectionand Location in Water Pipelines. PhD thesis, Chongqing: ChongqingUniversity, 2007

[5] T. Aboulnasr, K. Mayyas, A robust variable step-size LMS-type algorithm: anal-ysis and simulations, IEEE Trans. Signal Process. 45 (3) (March 1997) 631-639.

[6] K. Mayyas, A variable step-size selective partial update LMS algorithm, Digit. Signal Process. 23 (1) (January 2013) 75-85. 